\title{
Rechtsprechung
}

\section{Zur Zulässigkeit der Entfernung von Kunstinstallationen in einem Museum}

\author{
BGH Urteil vom 21.2.2019 - ZR I 99/17
}

\section{ECLI:DE:BGH:2019:210219UIZR99.17.0}

- Der I. Zivilsenat des Bundesgerichtshofs hat auf die mündliche Verhandlung vom 29. November 2018 durch den Vorsitzenden Richter Prof. Dr. Koch, die Richter Prof. Dr. Schaffert, Prof. Dr. Kirchhoff, Feddersen und die Richterin Dr. Schmaltz

für Recht erkannt:

Die Revision gegen das Urteil des Oberlandesgerichts Karlsruhe - 6. Zivilsenat - vom 26. April 2017 wird als unzulässig verworfen, soweit sie sich dagegen richtet, dass das Berufungsgericht den auf Zurückverweisung an das Landgericht gerichteten Hauptantrag zurückgewiesen hat. Im Übrigen wird die Revision zurückgewiesen.

Die Kosten der Revision trägt die Klägerin.

Von Rechts wegen

\section{Tatbestand:}

[1] Die Klägerin ist eine international tätige Künstlerin. Die Beklagte ist eine Gebietskörperschaft des öffentlichen Rechts und betreibt die Kunsthalle Mannheim als Eigenbetrieb.

[2] Im Auftrag der Beklagten schuf die Klägerin ab dem Jahr 2006 für den Dach- und Kuppelbereich des Billing-Baus der Kunsthalle Mannheim die Lichtinstallation „PHaradise“. Ein schriftlicher Vertrag wurde hierüber nicht geschlossen. In einem von der Klägerin und dem damaligen Direktor der Kunsthalle unterzeichneten Leihschein vom 12. April 2007 ist das Werk auf das Jahr 2007 datiert und als "Dauerleihgabe“ für die Sammlung bezeichnet. In der Rubrik "Leihzeitraum" befindet sich die Eintragung „03.03.07 - nach Absprache“.

[3] Die technische Grundlage der Lichtinstallation „PHaradise" bildete in den Oberlichtern des Billing-Baus installierte Leuchtmittel (Langfeld-Leuchtstoffröhren). Diese wurden so gesteuert, dass sich zunächst die Kuppel mit einem hellen Licht füllte, welches sich anschließend in die beiden Seitenflügel ausbreitete. Aufgrund des Lichtverlaufs konnte der Eindruck einer "atmenden“ oder „pulsierenden“ Bewegung entstehen. Die Leuchtstoffröhren waren zudem mit unterschiedlichen Folien beschichtet, so dass das Licht an manchen Stellen einen wärmeren und an anderen Stellen einen kälteren Farbton aufwies.
[4] Ab dem Jahr 2010 sanierte die Beklagte das Dach des Billing-Baus und versah es mit moderner Klima- und Lichtschutztechnik. Im Zuge dieser Maßnahmen wurden spätestens 2013 die körperlichen Bestandteile der Lichtinstallation "PHaradise" vollständig demontiert und nicht wieder eingebaut.

[5] Die Klägerin sieht in der Entfernung ihres Werks eine Verletzung ihres Urheberrechts. Sie ist der Ansicht, ihr stehe sowohl ein vertraglicher als auch ein gesetzlicher Anspruch auf Erhaltung des Werks zu. Es sei auch ohne unzumutbaren Aufwand möglich, das Werk wieder in den renovierten Räumlichkeiten zu installieren. Die derzeitige Beleuchtungssituation sei eine unzulässige Bearbeitung ihres Werks. Hilfsweise verlangt sie Schadensersatz.

[6] Das Landgericht hat die Klage abgewiesen. Die Klägerin hat in der Berufungsinstanz zuletzt beantragt:

Den Rechtsstreit an das Landgericht zur weiteren Sachaufklärung und zur Beweisaufnahme gem. § 538Abs.2 Nr.1und 4 ZPO zurückzuverweisen.

Hilfsweise unter Abänderung des Urteils des Landgerichts Mannheim vom 23.Oktober 2015 die Beklagte wie folgt zu verurteilen:

I. Hauptantrag: Erhalt durch Reinstallation

Die Beklagte wird unter Androhung der gesetzlich vorgesehenen Zwangsmittel verurteilt,

- 1. das Werk "PHaradise“ an dem Billing-Bau der Kunsthalle Mannheim innerhalb einer vom Gericht zu bestimmenden angemessenen Frist (z.B. bis zum Ende des Monats März 2015) wieder zu errichten bzw. instand zu setzen und instand zu halten, indem

- die ursprüngliche Beleuchtung innerhalb der zentralen Kuppel des Billing-Baus und unter den beglasten Dächern der Seitenflügel des Billing-Baus wiederhergestellt wird, wie auf der Abbildung Ergänzung 2 ersichtlich, mit der (technischen) Maßgabe,

- dass das Licht im Inneren der Kuppel des Billing-Baus entsprechend der farbbestimmenden Vorgabe der Klägerin kaltes Licht ist (klares, helles „Weiß" zum Beispiel infolge der Anbringung einer hitzefesten, blauen Gelatine (Folie) um die Leuchtstoffröhren) und 
- das Licht unter den beglasten Dächern der Seitenflügel des Billing-Baus in warmem, gelblichem Licht ist (heller "gelblicher" Lichtton gemäß der Vorgabe der Klägerin zum Beispiel infolge der Anbringung einer hitzefesten, gelben Gelatine (Folie) um die Leuchtstoffröhren)

und der weiteren Maßgabe,

- dass nach der Vorgabe der Klägerin eine gegenläufige Intensivierung und Abschwächung dieser unterschiedlichen Lichter in den unterschiedlichen Gebäudeteilen (aus dem Inneren des Kuppelteils einerseits und dem Inneren der beglasten Dächer der Seitenflügel andererseits) möglich ist, damit eine als atmende Bewegung erscheinende Wirkung eintritt, sowie

- dass nach zeitlicher Vorgabe der Klägerin ab der Dämmerung bis zu einem Zeitpunkt am Morgen (Sonnenaufgang) eine sich zunehmend beruhigende Lichtbewegung durch das Verändern der Lichtintensität von der Kuppel zu den Außenenden der beglasten Dächer der Seitenflügel des Billing-Baus "fließend" erfolgen kann und wieder zurück von den Außenenden der beglasten Dächer der Seitenflügel zur Kuppel

und

2. das Werk nach der Wiedererrichtung in seiner Funktion als Lichtinstallation zur Beleuchtung des Billing-Baus der Kunsthalle Mannheim entsprechend der Beschreibung Abbildung 3 sowie der vorstehenden Ziffer 1 zu nutzen.

\section{Hilfsweise zu Ziffer I Schadensersatz}

Die Beklagte wird verurteilt, an die Klägerin einen angemessenen, vom Gericht der Höhe nach zu bestimmenden, den Betrag in Höhe von $90.000 €$ nicht unterschreitenden Schadensersatz für die Vernichtung des Werks „PHaradise“ nebst Zinsen zu zahlen.

[7] Die Berufung der Klägerin ist ohne Erfolg geblieben (OLG Karlsruhe, ZUM-RD 2017, 600). Mit der vom Senat zugelassenen Revision verfolgt die Klägerin ihre Berufungsanträge weiter.

\section{Entscheidungsgründe:}

[8] A. Das Berufungsgericht hat angenommen, ein Anspruch auf Erhaltung oder Wiederherstellung des Werks "PHaradise" stehe der Klägerin weder auf gesetzlicher noch auf vertraglicher Grundlage zu.

[9] Die Bestimmung des $\$ 14$ UrhG stehe der Vernichtung des Werks nicht entgegen, weil nach Abwägung das Interesse des Urhebers an der Fortexistenz seines mit einem Bauwerk unlösbar verbundenen Kunstwerks in aller Regel hinter dem Interesse des Eigentümers an der anderweitigen Nutzung seines
Grundstücks zurücktreten müsse. Dies gelte auch für Kunstmuseen, die zwar in besonderem Maße dazu dienten, Kunstwerke für die Nachwelt zu erhalten, jedoch ebenfalls ein berechtigtes Interesse daran hätten, die Gebäude und Ausstellungsflächen bei Bedarf an den aktuellen Stand der Museumstechnik anzupassen oder von Zeit zu Zeit für die Präsentation anderer Kunstwerke zu nutzen. Aufgrund der erforderlichen Sanierungsarbeiten habe die Beklagte Anlass gehabt, eine Neukonzeption ihres Gebäudes und insbesondere der Lichttechnik zu erwägen. Hierbei sei sie nicht verpflichtet gewesen, die Neugestaltung an den Interessen der Klägerin auszurichten. Aus den Ethischen Richtlinien für Museen des International Council of Museums (ICOM) ergebe sich nichts Abweichendes. Es könne nicht davon ausgegangen werden, dass die Reputation der Klägerin durch die Vernichtung Schaden erleide, weil die Demontage im Zusammenhang mit erforderlichen Sanierungsarbeiten erfolgt sei.

[10] Die Beklagte habe sich im Streitfall mit der Aufnahme des Werks der Klägerin nicht jeder späteren Neufestlegung dieses Grundstücksteils begeben. Ob ein Abwehranspruch im Falle der Entfernung eines Kunstwerks von außerordentlich hohem künstlerischen Rang bestehe, könne dahinstehen. Die Entfernung des Werks könne im Streitfall nicht als rechtsmissbräuchlich angesehen werden. Es lägen auch keine außergewöhnlichen Umstände vor, die eine Erhaltung mit Blick auf den Rang des Kunstwerks rechtfertigten.

[11] Ein Anspruch folge auch nicht aus einer vertraglichen Abrede. Es habe sich zwar nach dem Parteiwillen um eine permanente Installation gehandelt. Hieraus ergebe sich aber keine Pflicht der Beklagten, das Werk unter weitgehender Einschränkung ihrer Eigentümerbefugnisse für die Dauer des Urheberrechtsschutzes zu erhalten.

[12] Die infolge der Sanierung entstandene bauliche Situation stelle auch keine Bearbeitung des Werks dar, so dass auch unter diesem Gesichtspunkt kein Anspruch auf Wiederherstellung bestehe.

[13] Ein Anspruch auf Reinstallation ergebe sich auch nicht aus den Eigentumsrechten der Klägerin. Diese gewährten ihr keine Rechtsposition, die die Dispositionsbefugnis der Beklagten über die künftige Gebäudenutzung in Frage stelle. Da die Beklagte zur baulichen Veränderung zur dauerhaften Beseitigung des Werks der Klägerin berechtigt sei, habe die Klägerin auch keinen Anspruch auf Wiederaufbau oder zur erneuten Installation nach Fertigstellung der Bauarbeiten. Gleiches gelte für den Schadensersatzanspruch.

[14] B. Die gegen diese Beurteilung gerichtete Revision der Klägerin hat keinen Erfolg. Die Revision ist unzulässig, soweit sie sich dagegen richtet, dass das Berufungsgericht den auf Zurückverweisung an das Landgericht gerichteten Hauptantrag zurückgewiesen hat (dazu I). Die Klage ist zulässig (dazu II). Der hilfsweise geltend gemachte, auf Reinstallation gerichtete 
Klageantrag ist nicht nach § 97 Abs.1, § 14 UrhG (dazu III), § 97 Abs.1, § 23 UrhG (dazu IV) oder etwaigen Eigentumsrechten der Klägerin (dazu V) begründet. Ein solcher Anspruch hat auch keine vertragliche Grundlage (dazu VI). Mangels Pflichtverletzung liegen daher auch die Voraussetzungen für einen mit dem weiteren Hilfsantrag geltend gemachten Schadensersatzanspruch nicht vor.

[15] I. Die Revision ist als unzulässig zu verwerfen, soweit sie sich dagegen richtet, dass das Berufungsgericht den auf $\mathrm{Zu}$ rückverweisung an das Landgericht gerichteten Hauptantrag zurückgewiesen hat.

[16] 1. Die Revision ist nach § 552 Abs. 1 ZPO als unzulässig zu verwerfen, soweit sie nicht in der gesetzlichen Form begründet ist. Nach $\S 551$ Abs. 3 Nr. 2 Buchst. b ZPO muss die Revisionsbegründung, soweit die Revision darauf gestützt wird, dass das Gesetz in Bezug auf das Verfahren verletzt sei, die Tatsachen bezeichnen, die den Mangel ergeben.

[17] 2. Die Revision stützt sich auf eine Verletzung von $\S 538$ Abs. 2 S. 1 Nr. 1 ZPO. Danach darf das Berufungsgericht die Sache, soweit ihre weitere Verhandlung erforderlich ist, unter Aufhebung des Urteils und des Verfahrens an das Gericht des ersten Rechtszuges nur zurückverweisen, soweit das Verfahren im ersten Rechtszuge an einem wesentlichen Mangel leidet und auf Grund dieses Mangels eine umfangreiche oder aufwändige Beweisaufnahme notwendig ist, und eine Partei die Zurückverweisung beantragt. Die Revision hat keine Tatsachen bezeichnet, aus denen sich ergibt, dass das Berufungsgericht die Sache nach dieser Bestimmung an das Landgericht zurückverweisen musste. Insbesondere gibt sie nicht an, unter welchem wesentlichen Mangel das Verfahren im ersten Rechtszug leidet, aufgrund dessen eine umfangreiche oder aufwändige Beweisaufnahme notwendig ist.

[18] II. Die Klage ist zulässig, insbesondere ist der Klagegrund iSd § 253 Abs. 2 Nr. 2 ZPO hinreichend bestimmt angegeben.

[19] Die Klägerin stützt ihre Klage auf gesetzliche Ansprüche gem. § 97 Abs.1, § 14 UrhG sowie auf eine vertragliche Grundlage. Hierbei handelt es sich um unterschiedliche Klagegründe und damit verschiedene Streitgegenstände (vgl. BGH, Urteil vom 24. Januar 2013 - I ZR 60/11, GRUR 2013, 397 Rn. 13 = WRP 2013, 499 - Peek \& Cloppenburg III). Gem. § 253 Abs. 2 Nr. 2 ZPO muss die Klageschrift die bestimmte Angabe des Gegenstandes und des Grundes des erhobenen Anspruchs enthalten. Die Klägerin hat daher klarzustellen, in welcher Reihenfolge sie die Streitgegenstände geltend macht. Diese Klarstellung kann noch in der Revisionsinstanz erfolgen (st. Rspr.; vgl. nur BGH, Urteil vom 22.März 2018 - I ZR 118/16, GRUR 2018, 1161 Rn. 23 = WRP 2018, 1329 - Hohlfasermembranspinnanlage, mwN).
[20] Die Klägerin hat in der Revisionsverhandlung klargestellt, dass sie ihre Ansprüche in erster Linie auf das Urheberrechtsgesetz stützt und in zweiter Linie auf vertragliche Ansprüche.

[21] III. Ohne Rechtsfehler hat das Berufungsgericht Ansprüche der Klägerin aus § 97 Abs. 1, § 14 UrhG verneint.

[22] 1.Wer das Urheberrecht widerrechtlich verletzt, kann von dem Verletzten nach § 97 Abs. 1 S. 1 Fall 1 UrhG auf Beseitigung der Beeinträchtigung in Anspruch genommen werden. Wer die Handlung vorsätzlich oder fahrlässig vornimmt, ist dem Verletzten nach $\S 97$ Abs. 2 S. 1 UrhG zum Ersatz des daraus entstehenden Schadens verpflichtet. Nach § 14 UrhG hat der Urheber das Recht, eine Entstellung oder eine andere Beeinträchtigung seines Werks zu verbieten, die geeignet ist, seine berechtigten geistigen oder persönlichen Interessen am Werk zu gefährden. Im Falle einer Verletzung dieses Rechts durch Bearbeitung oder Vernichtung eines Werks kommen Ansprüche auf Wiedererrichtung des Werks unter dem Gesichtspunkt der Beseitigung der Beeinträchtigung (im Falle der Bearbeitung) oder des Schadensersatzes in Form der Naturalrestitution (im Falle der Vernichtung) in Betracht.

[23] 2. Die Revision wendet sich nicht gegen die für sie günstige Annahme des Berufungsgerichts, es handele sich bei der streitgegenständlichen Installation um ein Werk iSv § 2 Abs. 1 Nr. 4, Abs. 2 UrhG. Rechtsfehler sind insoweit auch nicht ersichtlich.

[24] 3. Das Berufungsgericht hat die Deinstallation des Werks der Klägerin zu Recht am Maßstab des § 14 UrhG gemessen.

[25] a) Nach § 14 UrhG hat der Urheber das Recht, eine Entstellung oder eine andere Beeinträchtigung seines Werks zu verbieten, die geeignet ist, seine berechtigten geistigen oder persönlichen Interessen am Werk zu gefährden. Diese Vorschrift ist Ausdruck des Urheberpersönlichkeitsrechts, das den Schutz des geistigen und persönlichen Bandes zwischen Urheber und Werk zum Gegenstand hat (vgl. Begründung des Entwurfs eines Gesetzes über Urheberrecht und verwandte Schutzrechte, BTDrs. IV/270, S. 45; Dietz/Peukert in Schricker/Loewenheim, Urheberrecht, 5. Aufl., § 14 UrhG Rn. 5).

[26] b) Die Frage, ob die Vernichtung des Werks eine Beeinträchtigung iSd § 14 UrhG darstellt, ist umstritten.

[27] aa) Unter Hinweis darauf, dass § 14 UrhG das Interesse des Urhebers am Fortbestand des unverfälschten Werks, nicht aber das Interesse des Urhebers an der Existenz des Werks als solchem schütze, wird die Anwendung dieser Vorschrift auf die Vernichtung des Werks vielfach verneint (vgl. KG, GRUR 1981, 742; OLG Schleswig, ZUM 2006, 426, 427 [juris Rn. 9]; LG München I, FuR 1982, 510, 513; LG Hamburg, GRUR 2005, 672, 674 [juris Rn. 33]; Bullinger in Wandtke/Bullinger, Urheberrecht, 4. Aufl., § 14 UrhG Rn. 22 bis 24; Dietz/Peukert in Schricker/Loewenheim 
aaO § 14 UrhG Rn. 21; Dustmann in Fromm/Nordemann, Urheberrecht, 12. Aufl., § 14 UrhG Rn. 32 f.; B. Goldmann GRUR 2005, $639,643)$

[28] bb) Nach anderer Ansicht ist die Vernichtung eines Werkoriginals als schärfste Form der Beeinträchtigung iSd § 14 UrhG anzusehen. Sie verletze das Interesse des Urhebers, durch sein Werk auf den kulturellen oder gesellschaftlichen Kommunikationsprozess einzuwirken und im Werk fortzuleben (vgl. Dreyer in Dreyer/Kotthoff/Meckel/Hentsch, Urheberrecht, 4.Aufl., § 14 UrhG Rn. 50; Kroitzsch/Götting in Möhring/Nicolini, Urheberrecht, 4. Aufl., § 14 UrhG Rn. 24; Schulze in Dreier/Schulze, UrhG, 6. Aufl., § 14 Rn. 27 f.; Schack Urheber- und Urhebervertragsrecht, 8. Aufl. Rn. 397; ders. Kunst und Recht, Bildende Kunst, Architektur, Design und Fotografie im deutschen und internationalen Recht, 3. Aufl. Rn. 185; Dietz Das Droit Moral des Urhebers im neuen französischen und deutschen Urheberrecht, 1968, S.112; Schilcher Der Schutz des Urhebers gegen Werkänderungen, 1989, S.83 ff.; Schöfer Die Rechtsverhältnisse zwischen dem Urheber eines Werks der bildenden Kunst und dem Eigentümer des Originalwerks, 1984, S.139 f.; v. Waasen Das Spannungsverhältnis zwischen Urheberrecht und Eigentum im deutschen und ausländischen Recht, 1994, S. 151 ff.; Movsessian UFITA 95 (1983) S. 77, 85; Richard/Junker GRUR 2007, 18, 24; Samson UFITA 47 (1966) S. 1, 37).

[29] cc) Der Senat stimmt der letztgenannten Auffassung zu.

[30] (1) Nach seinem Wortlaut und seiner Systematik erfasst \$14 UrhG die Vernichtung des Werks. Zwar mag die in \$14 UrhG zunächst genannte Entstellung den Fortbestand des Werks voraussetzen. Bei der Entstellung handelt es sich aber nur um einen besonderen Fall der in §14 UrhG weiter genannten Beeinträchtigung des Werks. Das allgemeine Sprachverständnis steht der Annahme nicht entgegen, dass es sich bei der Vernichtung um einen weiteren Fall der Beeinträchtigung des Werks handelt. Soweit gegen die Anwendung des §14 UrhG auf die Werkvernichtung eingewandt wird, schon dem Wortsinn nach stelle eine Vernichtung keine Beeinträchtigung im Sinne dieser Vorschrift dar, weil die Beeinträchtigung ein Weniger gegenüber der Vernichtung sei (Schmelz GRUR 2007, 565, 568), liegt dem ein zu enges Wortverständnis zugrunde. Ist die in \$14 UrhG genannte andere Beeinträchtigung der tatbestandliche Oberbegriff und die gleichfalls genannte Entstellung lediglich ein Anwendungsfall dieses Oberbegriffs, steht das Sprachverständnis der Einbeziehung der Vernichtung in den Begriff der sonstigen Beeinträchtigung nicht entgegen.

[31] (2) Die Gesetzgebungsmaterialien stehen der Annahme nicht entgegen, dass nach $\$ 14$ UrhG die Vernichtung eines Werks verboten sein kann. In der Begründung zum Regierungsentwurf eines Urheberrechtsgesetzes heißt es zwar, es erscheine nicht angebracht, in das Gesetz ein Vernichtungsverbot für Werke der bildenden Künste aufzunehmen, soweit an ihrer Erhaltung ein öffentliches Interesse besteht; die Erhaltung kulturell wertvoller Kunstwerke sei nicht Aufgabe des privatrecht- lichen Urheberrechts, sondern des zum Gebiet des öffentlichen Rechts gehörenden Denkmalschutzes (BT-Drs. IV/270, S. 45). Dieser Begründung ist jedoch allein zu entnehmen, dass ein öffentliches Interesse an der Erhaltung eines Werks der bildenden Künste nach §14 UrhG kein Vernichtungsverbot begründen soll. Damit ist nicht gesagt, dass auch die durch §14 UrhG geschützten geistigen und persönlichen Interessen des Urhebers an seinem Werk kein Vernichtungsverbot rechtfertigen können.

[32] (3) Der Zweck des §14 UrhG, die berechtigten geistigen oder persönlichen Interessen des Urhebers an seinem Werk zu schützen, spricht dafür, dass der Urheber nach dieser Bestimmung grundsätzlich auch eine Vernichtung seines Werks verbieten kann. Das Urheberpersönlichkeitsrecht kann durch die Vernichtung eines Werks in besonderer Weise betroffen sein, weil die Vernichtung das Fortwirken des Werks (als Ausdruck der Persönlichkeit seines Schöpfers) vereiteln oder erschweren kann. Durch die Vernichtung wird das geistige Band zwischen dem Urheber und seinem Werk durchschnitten (Erdmann in Festschrift Piper, 1996, S. 655, 674).

[33] (4) Weiter ist zu beachten, dass der potentielle Interessenkonflikt zwischen dem Eigentümer eines Werks und seinem Urheber grundrechtlichen Wertungen unterliegt. Handelt es sich um einen privaten Eigentümer, kann er sich auf sein Grundrecht nach Art. 14 Abs.1 GG berufen, wenn er mit seinem Eigentum nach Belieben verfahren (§903 S. 1 BGB), es etwa vernichten möchte. Die öffentliche Hand - im Streitfall: die Beklagte als Gemeinde - kann sich zwar nicht auf den Grundrechtsschutz des Art. 14 Abs. 1 GG berufen (vgl. BVerfGE 61, 82, 100 ff.). Soweit das Eigentum Gegenstand und Grundlage kommunaler Betätigung ist, genießt gemeindliches Eigentum aber den verfassungsrechtlichen Schutz der Garantie der kommunalen Selbstverwaltung des Art. 28 Abs.2 S. 1 GG (vgl. BVerwGE 97, 143 [juris Rn. 27]). Mit der Unterhaltung der städtischen Kunsthalle erfüllt die Beklagte die ihr nach Art. 3c Abs. 1 der Verfassung des Landes Baden-Württemberg als Gemeinde obliegende Pflicht, das kulturelle Leben zu fördern.

[34] Für den Urheber streitet die in Art. 5 Abs. 3 S. 1 GG verbürgte Kunstfreiheit, die nicht nur den Schaffensprozess („Werkbereich“), sondern auch die für die Begegnung mit der Kunst erforderliche Darbietung und Verbreitung des Kunstwerks („Wirkbereich“) schützt (vgl. BVerfGE 30, 173, 189 [juris Rn. 49] - Mephisto; BVerfGE 119, 1, 21 f. [juris Rn.63] - Esra, mwN).

[35] Diesen grundrechtlichen Wertungen kann im Falle der Vernichtung eines Werks Rechnung getragen werden, wenn die Vernichtung als Beeinträchtigung des Werks von §14 UrhG erfasst und damit die im Tatbestandsmerkmal der "berechtigten geistigen oder persönlichen Interessen" des Urhebers angelegte Interessenabwägung eröffnet ist.

[36] 4. Ohne Erfolg wendet sich die Revision gegen die vom Berufungsgericht zugunsten der Beklagten vorgenommene Interessenabwägung. 
[37] a) Das Berufungsgericht hat angenommen, die Interessen des Urhebers am Fortbestehendes Werks müssten bei Werken der Baukunst, bei mit Bauwerken unlösbar verbundenen Werken oder sonst grundstücksbezogenen Kunstwerken in aller Regel hinter den Interessen des Eigentümers an der anderweitigen Nutzung oder Bebauung des Grundstücks und der damit verbundenen Zerstörung oder Entfernung des Werks zurückstehen. Dem Interesse des Urhebers sei in solchen Fällen in der Weise Rechnung zu tragen, dass ihm die Möglichkeit der Dokumentation des Werks vor seiner Zerstörung gegeben werde. Diese Grundsätze würden auch für Museen als Eigentümer von Werkstücken gelten, weil diese ein berechtigtes Interesse an baulichen Veränderungen der Ausstellungsflächen und Umgestaltungen der Ausstellungen für die Präsentation anderer Kunstwerke hätten.

[38] Im vorliegenden Fall überwiege das Interesse der Beklagten an der Umgestaltung des Gebäudeteils. Die streitgegenständliche Installation im Dachbereich beanspruche Flächen, die zum einen als Raum für Gebäudekunst in Betracht kommen und zum anderen für die lichttechnische Gebäudeausstattung relevant sein könnten. Die Klägerin habe deshalb damit rechnen müssen, dass die Beklagte bei einer lichttechnischen Neukonzeption des Gebäudeteils auch das Werk entfernt. Bei der von der Beklagten gewählten Neukonzeption sei ein Erhalt des Werks der Klägerin nicht möglich gewesen und die Beklagte sei auch nicht verpflichtet, die Neugestaltung an den Interessen der Klägerin auszurichten. Die Klägerin habe nur vorgetragen, dass eine Reinstallation des Werks angepasst an die neuen Gegebenheiten mit moderneren Mitteln möglich gewesen sei, nicht aber die Reinstallation in der ursprünglichen Form. Die Installation der Klägerin habe keine herausragende Bedeutung. Die Reputation der Klägerin erleide durch die Vernichtung keinen Schaden. Die Beklagte habe den vor der Anbringung ungenutzten Dachbereich auch nicht dazu bestimmt, auf Dauer für das Kunstwerk zur Verfügung zu stehen. Diese Beurteilung hält der rechtlichen Nachprüfung stand.

[39] b) Bei der im Rahmen des § 14 UrhG vorzunehmenden Interessenabwägung ist auf Seiten des Urhebers insbesondere zu berücksichtigen, ob es sich bei dem vernichteten Werk um das einzige Vervielfältigungsstück des Werks handelte, oder ob von dem Werk weitere Vervielfältigungsstücke existieren. Ferner ist zu berücksichtigen, welche Gestaltungshöhe das Werk aufweist und ob es ein Gegenstand der zweckfreien Kunst ist oder als angewandte Kunst einem Gebrauchszweck dient (vgl. Erdmann in Festschrift Piper, 1996, S. 655, 674; Schack Kunst und Recht aaO Rn. 185).

[40] Auf Seiten des Eigentümers können, etwa wenn ein Bauwerk oder Kunst in oder an einem solchen betroffen ist, bautechnische Gründe oder das Interesse an einer Nutzungsänderung von Bedeutung sein (vgl. BGH, Urteil vom 19. März 2008 - I ZR166/05, GRUR 2008, 984 Rn. 38 f.= WRP 2008, 1440 - St. Gottfried; Dietz/Peukert in Schricker/Loewenheim aaO § 14 UrhG Rn. 39 f.; Schulze in Dreier/Schulze aaO § 14 Rn. 28; Sch- ack Urheber- und Urhebervertragsrecht aaO Rn. 399). Bei Werken der Baukunst oder mit Bauwerken unlösbar verbundenen Kunstwerken werden die Interessen des Eigentümers an einer anderweitigen Nutzung oder Bebauung des Grundstücks oder Gebäudes den Interessen des Urhebers am Erhalt des Werks in der Regel vorgehen, sofern sich aus den Umständen des Einzelfalls nichts anderes ergibt (vgl. Schack Kunst und Recht aaO Rn.189).

[41] Im Rahmen der Interessenabwägung kann sich weiter auswirken, ob der Eigentümer dem Urheber Gelegenheit gegeben hat, das Werk zurückzunehmen oder - wenn dies aufgrund der Beschaffenheit des Werks nicht möglich ist - Vervielfältigungsstücke hiervon anzufertigen (vgl. Ulmer Urheber- und Verlagsrecht, 3. Aufl., S. 220; Erdmann in Festschrift Piper, 1996, S. $655,674 \mathrm{f}$.$) .$

[42] Die in diesem Zusammenhang gebotene tatrichterliche Interessenabwägung ist durch das Revisionsgericht lediglich daraufhin zu überprüfen, ob Denkgesetze oder Erfahrungssätze verletzt sind und die für die Interessenabwägung des konkreten Streitfalls maßgeblichen Gesichtspunkte berücksichtigt und zutreffend gewichtet worden sind (vgl. BGH, Urteil vom 28. Juli 2016 - I ZR 9/15, BGHZ 211, 309 Rn. 36 - auf fett getrimmt).

[43] c) Die Revision wendet sich ohne Erfolg gegen die Annahme des Berufungsgerichts, das Interesse des Urhebers am Fortbestehen eines mit einem Bauwerk unlösbar verbundenen Kunstwerks trete in aller Regel hinter die Interessen des Gebäudeeigentümers an einer anderweitigen Gebäudenutzung und einer damit verbundenen Zerstörung des Kunstwerks zurück. Zu der nach § 903 BGB dem Eigentümer zustehenden Befugnis, mit der Sache nach Belieben zu verfahren, gehört auch die Entscheidung über die Umgestaltung oder anderweitige Nutzung eines Gebäudes (vgl. Schulze in Dreier/Schulze aaO $\S 14$ Rn. 28; Schack Kunst und Recht aaO Rn. 195; von UngernSternberg in Weller/Kemle/Lynen, Des Künstlers Rechte - die Kunst des Rechts, 2007, S. 47, 59). Anders als bei zerstörungsfrei entfernbaren Kunstwerken wäre dieses Recht völlig aufgehoben, wenn der Urheber einer mit einem Gebäude unlösbar verbundenen Installation deren Entfernung dauerhaft untersagen könnte. Duldet ein Gebäude- oder Grundstückseigentümer die Installation eines solchen Werks, willigt er typischerweise nicht in eine so umfassende und sehr weit in die Zukunft reichende Beschränkung seiner Eigentümerbefugnisse ein. Dem Künstler steht demgegenüber die Möglichkeit offen, eine Erhaltungspflicht entweder schuldrechtlich zu vereinbaren oder auf der Einräumung einer beschränkt persönlichen Dienstbarkeit iSv § 1090 BGB zu bestehen, durch die er sich gegen eine spätere Entfernung des Kunstwerks durch Rechtsnachfolger des Eigentümers absichern kann (vgl. Schack Kunst und Recht aaO Rn. 196).

[44] d) Entgegen der Ansicht der Revision hat das Berufungsgericht dem Umstand zutreffend Rechnung getragen, dass die Beklagte als Gemeinde der öffentlichen Hand zuzurechnen ist. 
[45] Betroffen ist hier nicht der im Falle von Bauwerken typische Konflikt zwischen dem Eigentümer des Bauwerks und dem beauftragten Architekten (vgl. dazu BGH, GRUR 2008, 984 Rn. 25 bis 39 - St. Gottfried; BGH, Beschluss vom 9.November 2011 - I ZR 216/10, GRUR 2012, 172), sondern das Verhältnis zwischen einem Kunstmuseum der öffentlichen Hand und der Schöpferin eines zweckfreien Kunstwerks. Die Annahme des Berufungsgerichts, (auch) ein Kunstmuseum der öffentlichen Hand könne ein Interesse an einer Änderung der Museumsgebäude und der Ausstellungsflächen haben, lässt keine Rechtsfehler erkennen. Die Anerkennung eines urheberrechtlichen Verbots der Entfernung von mit einem Gebäude unlösbar verbundenen Installationen hinderte die Museen dauerhaft an der Umgestaltung von Ausstellungen und Museumsgebäuden. Museen können ihren kulturellen Auftrag nur erfüllen, wenn sie sich an veränderte kulturelle oder gesellschaftliche Bedürfnisse durch Änderungen der Gebäude und Ausstellungskonzepte anpassen können.

[46] e) Die Revision rügt vergeblich die Annahme des Berufungsgerichts, die Beklagte habe die Neugestaltung des Museums nicht an den Interessen der Klägerin am Werkerhalt ausrichten müssen.

[47] aa) Nach der Rechtsprechung des Bundesgerichtshofs muss im Rahmen der bei §14 UrhG erforderlichen Interessenabwägung bei Änderungen eines Werks der Baukunst nicht geprüft werden, ob andere Planungsalternativen zu einer geringeren Beeinträchtigung der Interessen des Urhebers geführt hätten. Zwar muss der Eigentümer eines urheberrechtlich geschützten Bauwerks bei dessen Veränderung grundsätzlich eine den betroffenen Urheber in seinen urheberpersönlichkeitsrechtlichen Interessen möglichst wenig berührende Lösung suchen. Hat er sich jedoch für eine bestimmte Planung entschieden, so geht es im Rahmen der Interessenabwägung nur noch darum, ob dem betroffenen Urheber die geplanten konkreten Änderungen des von ihm geschaffenen Bauwerks zuzumuten sind. Ob daneben noch andere, den Urheber gegebenenfalls weniger beeinträchtigende Lösungen denkbar sind, ist hierfür nicht von entscheidender Bedeutung $(\mathrm{BGH}$, Urteil vom 31. Mai 1974 - I ZR10/73, BGHZ 62, 331, 338 [juris Rn. 36] Schulerweiterung; BGH, GRUR 2008, 984 Rn. 39 - St. Gottfried; von Ungern-Sternberg aaO 47, 59).

[48] bb) Diese Grundsätze gelten erst recht, wenn es - wie im vorliegenden Fall - nicht um die Änderung eines Werks der Baukunst, sondern um die mit seiner Zerstörung verbundene Entfernung eines ortsgebundenen Kunstwerks aus einem Gebäude im Zuge seiner baulichen Umgestaltung geht. Anders als bei der Veränderung eines Werks der Baukunst verfälscht die Vernichtung einer mit dem Gebäude verbundenen Installation nicht die Gestalt des Werks, in der es nach außen tritt. Die Veränderung des Werkstücks berührt stets das Interesse des Urhebers an der Entscheidung darüber, wie das Werk an die Öffentlichkeit treten soll (BGH, Urteil vom 1. Oktober 1998 - I ZR104/96, GRUR 1999, 230, 232 [juris Rn. 30] - Treppenhaus- gestaltung). Bei einer Werkzerstörung besteht hingegen keine Gefahr der Werkverfälschung, weil das Werk nicht mehr wahrnehmbar ist.

[49] cc) Die von der Revision als übergangen gerügte Behauptung der Klägerin, eine Installation des Werks nach Abschluss der Sanierungsarbeiten sei ohne Änderung des Werks möglich, wirkt sich im Rahmen der Interessenabwägung nicht entscheidungserheblich aus. Die Möglichkeit der Wiedererrichtung des Werks lässt das in die Abwägung einzustellende Interesse der Klägerin nicht in einem Maße erstarken, dass der Beklagten eine Änderung ihres Nutzungskonzepts zu untersagen wäre.

[50] f) Ästhetische Interessen allein berechtigen den Eigentümer in der Regel nicht zur Umgestaltung eines Kunstwerks (vgl. BGH, GRUR 1999, 230, 232 [juris Rn. 30] - Treppenhausgestaltung; von Ungern-Sternberg aaO 47, 60). Es kann offenbleiben, ob dies auch für die mit ihrer Zerstörung einhergehende Entfernung einer solchen Installation im Rahmen eines Gebäudeumbaus gilt. Nach den Feststellungen des Berufungsgerichts erfolgte die Entfernung im Streitfall nicht allein aus ästhetischen Gründen, sondern weil das Dach völlig umgestaltet und die lichttechnische Ausstattung neu konzipiert werden sollte.

[51] g) Die Revision wendet sich vergeblich gegen die Gewichtung der weiteren schutzwürdigen Interessen der Beklagten an der Entfernung der Installation. Sie rügt erfolglos, das Berufungsgericht habe nicht berücksichtigt, dass das Kunstwerk der Klägerin keine für museale Zwecke genutzten Flächen beanspruche, weil der hierfür genutzte Bereich vor der Installation keine Funktion gehabt und folglich die Beklagte diesen Bereich bewusst der Kunst gewidmet habe.

[52] Das Berufungsgericht hat diesen Vortrag gesehen, ihn aber im Hinblick darauf nicht für durchgreifend gehalten, dass das Kunstwerk in die lichttechnische Gebäudeausstattung eingreift und deshalb für die weiteren Ausstellungsflächen relevant ist. Diese Würdigung, insbesondere die Berücksichtigung der lichttechnischen Bedeutung des Installationsorts für die anderen Museumsbereiche, unterliegt keinen revisionsrechtlichen Bedenken.

[53] h) Ohne Erfolg wendet sich die Revision gegen die Würdigung der schutzwürdigen Interessen der Klägerin am Erhalt des Werks durch das Berufungsgericht.

[54] Vergeblich macht die Revision geltend, die Klägerin habe nicht mit einer willkürlichen einseitigen Entfernung der Installation zum Zwecke der Veränderung der lichttechnischen Konzeption des Gebäudes rechnen müssen, weil es bis zur streitgegenständlichen Installation überhaupt kein elektrisches Licht in dem Bereich gegeben habe. Hiermit setzt sie lediglich ihre eigene Würdigung der Umstände an die Stelle der rechtsfehlerfreien tatrichterlichen Würdigung. 
[55] i) Ohne Erfolg beanstandet die Revision die Feststellungen des Berufungsgerichts zum künstlerischen Rang des Kunstwerks.

[56] Die Annahme des Berufungsgerichts, das Werk sei nicht von herausragender künstlerischer Bedeutung, weil es bereits im Jahr 2006 ortspezifische Lichtinstallationen internationaler Künstler gegeben habe und die streitgegenständliche Installation in der kunsthistorischen Forschung keine Beachtung gefunden habe, begegnet keinen revisionsrechtlichen Bedenken. Die Revision ersetzt auch hier lediglich die tatrichterliche Würdigung durch ihre eigene Sichtweise.

[57] Das Berufungsgericht war entgegen der Auffassung der Revision weiterhin nicht verpflichtet, für die Beurteilung des künstlerischen Rangs ein Sachverständigengutachten einzuholen, sondern vermochte aufgrund eigener Sach-kunde zu entscheiden. Die Mitglieder eines fachspezifischen Spruchkörpers haben regelmäßig hinreichenden Sachverstand, um die Schutzfähigkeit und Eigentümlichkeit eines Werks der bildenden Kunst zu beurteilen. Dies gilt jedenfalls dann, wenn der Anspruchsteller sich für den behaupteten Rang des Werks auf dessen Eindruck und Form und nicht auf die Beurteilung in der Kunstwelt stützt (vgl. in diesem Sinn zu Bauwerken: BGH, Urteil vom 29. März 1957 - I ZR236/55, BGHZ 24, 55, 67 f. [juris Rn. 27] - Ledigenheim; BGH, GRUR 2008, 984 Rn. 20 - St. Gottfried; OLG Karlsruhe, GRUR-RR 2013, 423, 427 [juris Rn. 38]; OLG Stuttgart, GRUR-RR 2011, 56, 58 [juris Rn. 27]; Schulze in Dreier/ Schulze aaO § 2 Rn. 60; anders zu Musikwerken: BGH, Urteil vom 16. April 2015 - I ZR225/12, GRUR 2015, 1189 Rn. 59 ff. = WRP 2015, 1507 - Goldrapper). Der von der Revision als übergangen gerügte Beweisantrag der Klägerin stützte sich maßgeblich auf die Wirkung des Kunstwerks auf den Betrachter, seine Funktion innerhalb des Gebäudes sowie die von der Beklagten in der Vergangenheit getätigten Aussagen hierzu. Diese Aspekte konnte das Berufungsgericht aus eigener Sachkunde beurteilen. Die Revision legt auch nicht konkret dar, von welchen falschen oder streitigen entscheidungserheblichen Tatsachen das Berufungsgericht bei seiner Beurteilung des künstlerischen Rangs ausgegangen sein soll.

[58] IV. Die derzeitige bauliche Situation stellt keine Bearbeitung des Werks der Klägerin dar, so dass auch eine Verletzung des § 23 UrhG ausscheidet. Für eine Bearbeitung iSd § 23 UrhG müssen schutzfähige Merkmale des Originalwerks verändert werden. Die schutzfähigen Merkmale der Installation sind vorliegend vernichtet, weshalb insoweit § 14 UrhG vorgeht.

[59] V. Das Berufungsgericht hat ferner zutreffend angenommen, dass der Klägerin die geltend gemachten Ansprüche auch nicht aus eigentumsrechtlichen Erwägungen zustehen. Es kann offenbleiben, ob die Klägerin Eigentümerin einzelner Bestandteile der Installation gewesen ist. Hieraus folgt jedenfalls kein Anspruch auf eine bestimmte Nutzung dieser Bestandteile im Gebäude der Beklagten.
[60] Vl. Ohne Erfolg wendet sich die Revision gegen die Beurteilung des Berufungsgerichts, der hilfsweise geltend gemachte Anspruch auf Reinstallation habe keine vertragliche Grundlage.

[61] 1. Das Berufungsgericht hat ausgeführt, bei Auftragserteilung habe festgestanden, dass das Objekt „PHaradise“ eine permanente Installation habe sein sollen. Hieraus ergebe sich allerdings keine Verpflichtung der Beklagten, das Werk unter weitgehender Einschränkung ihrer Eigentümerbefugnisse für die Dauer des Urheberrechtsschutzes zu unterhalten. Der Sinngehalt des Adjektivs "permanent" sei nach dem im Museumsbereich üblichen Sprachgebrauch dahin zu verstehen, dass die Ausstellung oder Installation im Unterschied zur Sonderausstellung nicht auf bestimmte Zeit angelegt sei. Daraus ergebe sich mit Blick auf die Interessenlage der Parteien nicht, dass die Beklagte auf alle Zeit an einer endgültigen Demontage gehindert sei. Dass beide Parteien eine Demontage für möglich gehalten hätten, ergebe sich auch aus der im Leihschein enthaltenen Angabe „03.03.07 - nach Absprache“. Die Darlegung der Klägerin, die Parteien hätten das Werk als festen und dauerhaften Bestandteil der Kunsthalle bestimmt, sei unsubstantiiert, weil die Klägerin selbst vorgetragen habe, dass zu keinem Zeitpunkt die Frage im Raum gestanden habe, ob, wann oder unter welchen Umständen das Werk dauerhaft deinstalliert und der Öffentlichkeit nicht mehr zugänglich sein solle. Mangels ausdrücklicher Absprache habe die Klägerin aufgrund der Interessenlage der Parteien nicht davon ausgehen dürfen, dass das Werk nach einer erforderlichen Sanierungsmaßnahme wieder errichtet werde. Diese Beurteilung hält der rechtlichen Nachprüfung stand.

[62] 2. Die Auslegung von Individualvereinbarungen ist grundsätzlich Sache des Tatrichters. Dessen Auslegung unterliegt im Revisionsverfahren nur einer eingeschränkten Überprüfung im Hinblick darauf, ob gesetzliche Auslegungsgrundsätze, Denkgesetze oder Erfahrungssätze verletzt sind oder ob die Auslegung auf Verfahrensfehlern beruht, etwa weil wesentliches Auslegungsmaterial unter Verstoß gegen Verfahrensvorschriften außer Acht gelassen worden ist (st. Rspr.; vgl. BGH, Urteil vom 17. März 2011 - I ZR 93/09, GRUR 2011, 946 Rn. 17 f. = WRP 2011, $1302-\mathrm{KD}$; Urteil vom 18. Oktober 2017 - I ZR 6/16, GRUR 2018, 297 Rn. 32 = WRP 2018, 551 - media control, jeweils $\mathrm{mwN}$ ). Leidet die tatrichterliche Auslegung an solchen revisionsrechtlich beachtlichen Rechtsfehlern, bindet sie das Revisionsgericht nicht. Bei der Auslegung sind in erster Linie der von den Parteien gewählte Wortlaut und der dem Wortlaut zu entnehmende objektiv erklärte Parteiwille zu berücksichtigen. Weiter gilt das Gebot der nach beiden Seiten hin interessengerechten Auslegung und der Berücksichtigung des durch die Parteien beabsichtigten Zwecks des Vertrags (BGH, GRUR 2011, 946 Rn. 18 - KD, mwN).

[63] 3. Ohne Erfolg wendet die Revision ein, die vom Berufungsgericht vorgenommene Auslegung verstoße gegen den Grundsatz, in erster Linie den von den Parteien gewählten Wortlaut zu berücksichtigen, weil die Interpretation des Wortes „permanent" sinnwidrig sei. 
[64] Das Berufungsgericht ist vom Wortlaut der Vereinbarung ausgegangen und hat zugunsten der Klägerin unterstellt, dass die Parteien über den im Leihschein niedergelegten Wortlaut hinaus von einer permanenten Installation gesprochen haben. Es hat sodann den Sinngehalt dieser Formulierung in Hinblick auf den hier vorliegenden musealen Kontext in der Weise ausgelegt, dass er iSv "nicht auf bestimmte Zeit angelegt" zu verstehen sei. Diese Auslegung ist nicht wortsinnwidrig. Das Wort "permanent" bedeutet zwar "dauerhaft". Dieser Begriff enthält jedoch keine Aussage darüber, wann und unter welchen Voraussetzungen der Dauerzustand beendet werden kann. Im allgemeinen Sprachgebrauch kann auch ein permanenter Zustand zu einem späteren Zeitpunkt beendet werden.

[65] Die Revision rügt weiter vergeblich, dass das Berufungsgericht nicht den von der Klägerin vorgetragenen, in der Kunstwelt verbreiteten Begriffsinhalt berücksichtigt habe. Das Berufungsgericht hat sich mit der Bedeutung des Begriffs im musealen Bereich ausdrücklich befasst. Rechtsfehler sind ihm hierbei nicht unterlaufen.

[66] 4. Ohne Erfolg macht die Revision geltend, das Berufungsgericht habe gegen das Gebot der nach beiden Seiten interessengerechten Auslegung verstoßen, indem es einseitig den Interessen der Beklagten zum Durchbruch verholfen und verkannt habe, dass die Klägerin nicht mit einer Zerstörung des Kunstwerks rechnen musste.

[67] Das Gebot der nach beiden Seiten interessengerechten Auslegung erfordert, die zum Zeitpunkt des Vertragsschlusses erkennbaren wechselseitigen Interessen zu berücksichtigen und die Abrede auf einen vertretbaren Sinngehalt zurückzuführen (vgl. BGH, Urteil vom 16. Februar 2012 - I ZR 150/10, TranspR 2012, 148 Rn. 37 mwN). Die vom Berufungsgericht vorgenommene Auslegung genügt diesen Anforderungen, indem sie die zum Zeitpunkt der Vereinbarung erkennbare Interessenlage der Parteien darstellt und hierbei auch auf die Interessen der Klägerin eingeht. Das Berufungsgericht kommt zu dem vertretbaren Ergebnis, dass ein unentgeltlicher Leihvertrag nur eine Berechtigung und keine Verpflichtung des Entleihers zur Nutzung der Sache begründet und ein etwaiger anderweitiger Wille der Klägerin für die Beklagte beim Vertragsschluss nicht erkennbar war. Hierbei hat das Berufungsgericht auch den Vortrag der Klägerin zur Kenntnis genommen, ein moderner Künstler rechne bei einer permanenten Installation nicht mit deren baldiger Entfernung und gehe erst recht nicht davon aus, dass eine nach Beendigung von Baumaßnahmen mögliche Neuinstallation unterbleibe. Das Berufungsgericht hat diesen Vortrag nicht nur im Urteilstatbestand erwähnt, sondern es hat sich hiermit auch in den Entscheidungsgründen befasst.

[68] 5. Ohne Erfolg macht die Revision geltend, das Berufungsgericht habe nicht berücksichtigt, dass der Klägerin unter Hinweis auf ein als Anlage K 21 bezeichnetes Schreiben die Erhaltung der Installation zugesagt worden sei.
[69] Der Geltendmachung eines Verstoßes des Berufungsgerichts gegen das Gebot rechtlichen Gehörs (Art. 103 Abs. 1GG) steht der Grundsatz der Subsidiarität entgegen. Die Klägerin hat den als übergangen gerügten Vortrag bereits in erster Instanz gehalten. Das Landgericht hat sich mit diesem Vortrag nicht befasst. Die Revision hat nicht dargelegt, dass die Klägerin in der Berufungsinstanz die Nichtberücksichtigung dieses Vortrags durch das Landgericht gerügt hat. Damit hat sie gegen das Gebot verstoßen, alle prozessualen Möglichkeiten auszuschöpfen, um eine Korrektur einer behaupteten Gehörsverletzung zu erwirken. Ihr ist es daher verwehrt, die behauptete Gehörsverletzung erstmals in der Revisionsinstanz zu rügen (vgl. BGH, Beschluss vom 17. März 2016 - IX ZR 211/14, NJW-RR 2016, 699 Rn. 4).

[70] 6. Die Revision macht vergeblich geltend, das Berufungsgericht habe rechtsfehlerhaft geprüft, ob eine Erhaltungspflicht aus dem Vertrag bestehe; vielmehr sei die Frage der Befugnis zur Zerstörung ohne Rücksprache maßgeblich, für die der Leihschein eine Absprache vorsehe.

[71] Die Klägerin hat ihren vertraglichen Anspruch auf Erhalt des Werks auf eine vertragliche Verpflichtung zum Erhalt des Werks gestützt und nicht auf die Verletzung einer vertraglichen Verpflichtung, das Werk nur in Absprache mit der Klägerin zu demontieren. Sie kann in der Revisionsinstanz keinen neuen Streitgegenstand in den Rechtsstreit einführen. Im Übrigen könnte sich aus einem Verstoß gegen eine solche Verpflichtung jedenfalls kein Anspruch auf Erhalt des Werks ergeben.

[72] Danach ist die Revision, soweit sie unzulässig ist, zu verwerfen und im Übrigen mit der Kostenfolge aus § 97 Abs. 1 ZPO zurückzuweisen.

Koch - Schaffert - Kirchhoff - Feddersen - Schmaltz

\section{Vorinstanzen:}

LG Mannheim, Entscheidung vom 23.10.2015 - 7 O 70/15 OLG Karlsruhe, Entscheidung vom 26.04.2017 - 6 U 207/15 - 